\title{
Le poète au travail : La Grande Muraille. Journal de La Déchirure (1960-1965). L’apprentissage de la « réalité »
}

Lors du précédent colloque «Habiter le monde en poète. Henry Bauchau ou le livre en partage » organisé en mars 2007 par Catherine Mayaux et Myriam Watthee-Delmotte, et dont les actes ont paru en 2009 aux Presses Universitaires de Vincennes, j'ai voulu essayer de comprendre comment, dans l'œuvre poétique d'Henry Bauchau, exégèse de soi et exégèse du monde vont de conserve et comment le questionnement herméneutique se trouve au fondement d'une écriture de la présence au monde ${ }^{1}$. Geneviève Henrot, dans la «Lecture » qui accompagne Heureux les déliants, en 1995, analyse également l'évolution $\mathrm{du}$ poète et sa «fascination grandissante [...] pour l'épaisseur du quotidien, pour la circonstance de l'bic et nunc» même lorsqu'il s'agit d'un espace « revisité par la mémoire » ${ }^{2}$, après une période où «la perception est vive, mais mentale, spirituelle, intermittente », «la grève des sens donn[ant] ainsi la mesure du retranchement en soi-même que requièrent les nœuds de l'enfance $[. .].)^{3}$. Un changement s'opère, selon elle, au moment où "psychanalyse et création ont commencé à se féconder l'une l'autre $[\ldots] »^{4}$.

La Grande Muraille, le journal des années 1960-1965 pendant lesquelles Henry Bauchau écrit La Déchirure, correspond précisément à ce tournant et j'ai entrepris d'y suivre l'apprentissage, par l'écrivain, de la « réalité ». Ce qu'est cette « réalité » - de la « gloire du corps » ${ }^{5}$ à la « vie profonde »

\footnotetext{
${ }^{1}$ Aude Préta-de Beaufort, « Henry Bauchau, poète herméneute : exégèse de soi, exégèse du monde », dans Catherine Mayaux et Myriam Watthee-Delmotte (dir.), Henry Bauchau, écrire pour habiter le monde, Saint-Denis, Presses Universitaires de Vincennes, «L'Imaginaire du texte », 2009, pp. 39-48.

${ }^{2}$ Geneviève Henrot, « Lecture », dans HD, p. 354.

${ }^{3}$ Ibid., p. 347.

${ }^{4}$ Idem.

${ }^{5}$ GM, p. 393.
} 
-, de quelle façon lui « être fidèle $»^{6}$ et comment faire du journal l'atelier de «l'écriture du corps ${ }^{7}$, telles sont à peu près les questions que je me propose d'aborder, sans prétendre les épuiser ni épuiser avec elles les richesses d'un journal de presque cinq années.

\section{« Gloire du corps » et « vie profonde » : la réalité selon Henry Bauchau}

Il est frappant, et même touchant, de voir l'écrivain, alors qu'il est parvenu à l'âge mûr, consigner tout au long de son journal, son amour de la vitesse, le plaisir qu'il ressent à éprouver l'«aisance délicieuse » du corps dans l'exercice sportif maitrisé, en particulier le ski et la nage, son goût irrépressible de la chaleur et du soleil, la satisfaction qu'il retire de certaines promenades dans des paysages dont il admire la beauté :

Hier retour de Paris en sept heures et demie de route soit à plus de $80 \mathrm{kms}$ heure de moyenne ce qui me satisfait beaucoup. Le plaisir que j'en ai ressenti est vraiment comique. Le goût de la vitesse (dans la prudence) est une chose nouvelle en voiture. Je l'ai goûté tellement autrefois à cheval, en nageant, au saut à la perche, à skis. La voiture vient heureusement relayer tout cela.

Plaisir de traverser cette France verdoyante fleurie d'aubépines et de genêts. Gloire de juin qui est comme celle de la $20^{\mathrm{e}}$ année.

Plaisir de retrouver aujourd'hui Bergsrösli tout fleuri. (GM, 9 juin 1962, p. 207.)

Le premier bain vraiment chaud, l'allégresse du sel sur la peau, une joie de vagues et de gouttelettes.

La vue sur la montagne Sainte-Victoire et les allées successives au sud d'Aix.

$[\ldots]$

\footnotetext{
${ }^{6}$ Ibid., p. 252.

${ }^{7} \mathrm{HD}, \mathrm{p} .234$.
} 
Le souvenir d'une promenade pieds nus jusqu'à la petite anse au pied du phare de Camaret.

Je me suis jeté à l'eau ayant chaud et pendant une cinquantaine de mètres il m'a semblé retrouver ma forme d'autrefois et dominer l'eau avec une délicieuse aisance. (Ibid., 5 octobre 1963, p. 295.)

Le corps a envie de s'étendre, de jouir de la chaleur, et la conscience voudrait que je me mette au travail et renonce au plaisir d'être, d'être seul avec moi et avec tout, ayant délicieusement un peu trop chaud.

Je m'accorde successivement ces deux plaisirs. (Ibid., 13 mars 1965, p. 384.)

Attrait de cet automne si beau qu'il me semble jamais n'en avoir vu de pareil. Mardi durant les deux heures où j'aurais pu travailler, j'ai fait dans la vallée du Turbach une merveilleuse promenade avec L. Je ne puis le regretter car nous avons baigné un moment dans l'essentiel de l'automne et l'oubli des préoccupations courantes au moins durant quelques minutes où, assis sur un banc, nous avons regardé la vallée. (Ibid., 18 octobre 1962, p. 250.)

Loin d'être anecdotiques, ces épisodes possèdent souvent une dimension existentielle intense. Le sujet y vit des expériences de pleine présence au monde et à lui-même. Ainsi en août 1962 :

Forte sensation d'exister en m'asseyant sur la terrasse. Le soleil prend possession de mon torse et de mes épaules. Un lien s'est créé entre lui et le plexus qui semble s'ouvrir, fleurir un peu. Sous ma main gauche, je sens le mouvement du bras qui écrit et dans mon esprit passe un souvenir lointain des Ardennes et d'une route descendante vers la vallée de la Semois. (GM, p. 226.)

À la faveur de l'été, soleil et plexus «solaire» communiquent. La conscience du corps, comme dans certains exercices de méditation, s'aiguise et la concentration fait éprouver une participation d'ordre cosmique, tandis que d'autres pans de l'existence semblent près de se laisser ressaisir. 
Henry Bauchau évoque ailleurs, comme le Rousseau des Rêveries, des formes d'extase immanente, où le sujet entre en contact avec plus grand que lui, passe ses limites pour communiquer avec ce qui se laisse appréhender comme un Tout. La note de novembre 1962, dédiée à la beauté des mélèzes « glorieux ${ }^{8}$, s'articule autour du moment où le poète constate l'expansion du moi au sein d'un ensemble plus vaste que lui : "Je suis heureux là, mais d'un bonheur plus grand que moi et qui, incessamment, déborde $»^{9}$. À peine un an plus tard, la même expérience s'approfondit et précise son caractère d'extase immanente, dans la note du 25 octobre 1963 :

Les mélèzes : Je me suis couché la tête en bas dans la pente et je les ai regardés plonger dans le ciel. Moment non pas de bonheur, mais de tranquillité profonde, je n'existais plus pour moi, j'étais dans l'autre. Un autre infiniment plus grand et de cette noblesse limpide et passagère qui serre et dilate le cœur. (GM, p. 302.)

À la fois absolus et passagers, ces moments où le moi franchit ses propres limites pour éprouver intensément son appartenance à une dimension qui le dépasse restent étroitement liés à la réalité du monde sensible. Point culminant de la note de novembre 1962, l'extase du poète décline avec le jour :

Je me déplace, me mets à contre-jour, les arbres rayonnent plus fort, le soleil leur fait une bordure éblouissante, mais ils sont moins beaux ainsi, un point de perfection est dépassé. $[\ldots]$

L'enchantement se défait peu à peu, je sens le froid qui s'approche, le moment le plus parfait est dépassé. Je retrouve la terre étrangère alors qu'un instant auparavant elle était mienne, merveilleusement connue, familière. L'ombre et le froid gagnent, il faut partir. (GM, p. 254.)

Le «moment le plus parfait» reste inscrit dans un complexe de circonstances, de sensations, d'émotions, dont il dépend. Il ne s'agit pas de quitter l'immanence, mais de connaitre, au sein de la durée et du

\footnotetext{
${ }^{8}$ GM, 4 novembre 1962 , p. 253.

${ }^{9}$ Ibid., p. 254.
} 
mouvement de l'existence, des moments de communion et de pleine présence au monde :

Je suis compris dans cette splendeur verte et bleue, légèrement nuageuse et très feuillue. Je passe et en même temps je demeure dans cet instant plein et svelte comme le tronc d'un jeune arbre, dans cet instant qui passe, qui est déjà passé. (GM, 25 juillet 1961, p. 138.)

Ces expériences confirment à Henry Bauchau que le corps, entendu comme le lieu d'une présence totale du sujet au monde, n'est étranger ni à la vie psychique profonde ni à la vie de création.

C'est ce que semble enseigner le petit écureuil venu prendre vivement un morceau de biscuit dans la main du poète, un 26 mai 1960: sa sauvagerie et son corps palpitant paraissent manifester l'inconscient luimême et amènent une réflexion sur l'impossibilité de séparer « la réalité » et « la réalité intérieure » $(G M$, p. 64).

De la même façon, la contemplation du paysage communique avec les « couches plus profondes » qui sont celles de l'enfance, c'est-à-dire celles que l'analyse a explorées, et, partant, celles auxquelles ramène le roman en cours et que le journal accompagne. À la fin du mois d'août 1962, alors qu'il est allé retrouver les Jouve en Engadine, le paysage de la presqu'île de Chasté fait vivre au poète un moment d'extase analogue à ceux qu'il évoque en novembre 1962 ou en octobre 1963, mais noué à l'univers de la rêverie enfantine :

Les montagnes s'emmêlaient de longs et légers nuages et créaient des formes d'une fantaisie aérienne. On se retrouvait dans le monde de la légende enfantine, tout avait perdu sa pesanteur, on se perdait pour quelques instants dans quelque chose de divin, de tendre, de mélancolique car on percevait sa solitude. (GM, p. 240.)

En octobre 1963, une autre promenade, au cours de laquelle le poète s'étonne de la présence, en altitude, d'une truite «dans une petite mare sombre », confirme ce lien entre le paysage appréhendé et la matière psychique ancienne :

Ce lieu avait quelque chose du rêve et des étonnements de l'enfance, une sorte de déplacement du temps s'est opéré. 
Était-ce moi, encore moi tel que les autres actuellement me voient? Je retrouvais des couches plus profondes. (GM, p. 355.)

Le rêve du 28 mai 1964, relaté et analysé de façon assez développée ${ }^{10}$, donne à voir d'une autre manière qu'une véritable circulation existe entre le souci de la présence au monde et la matière psychique. Le poète identifie au monde et au cosmos le sourire qui s'empare extatiquement de lui dans son rêve. Mais ce sourire est aussi l'« intériorité la plus profonde » du moi : «Le sourire est le monde en moi faisant craquer mes parois sans pourtant sortir de moi-même $»^{11}$. L'expérience vécue ici d'une dilatation du moi aux proportions du monde à la faveur du rêve apparait comme la réciproque de l'expérience, plus souvent évoquée par le poète dans son journal, d'une fusion du moi dans le monde à la faveur de la perfection d'un paysage.

Quant à la vie de création, elle est elle aussi nouée à la nécessité d'embrasser la réalité et aux manifestations de la vie psychique profonde. Dans la note du $1^{\text {er }}$ avril 1965, après s'être à plusieurs reprises interrogé sur le risque de n'être pas «assez près de la réalité » ${ }^{12}$, Henry Bauchau fait du spectacle des montagnes et des glaciers qui forment devant lui une sorte de théâtre solennel l'occasion d'une méditation sur la parenté entre les structures de son imaginaire créateur et la poétique qu'il veut faire sienne d'une part, et celles des paysages qui lui sont familiers d'autre part :

Les deux sommets, unis par les épaules au-dessus d'une belle pente de nuages, cet équilibre, cette mesure dans l'immense, la nécessité et la fantaisie des courbes et des pentes se sont imprimés en moi. C'est par là que je résiste à ce qu'a de sec, d'abstrait et de désordonné une partie de l'art et de la littérature moderne. Il y a une présence en moi de ces deux puissantes assises, de ce mouvement de chaîne et de cette ascension solide. Je ne puis pas plus l'oublier, ni le nier que le glissement incessant du torrent en moi depuis dix ans bientôt. (GM, p. 390.)

\footnotetext{
${ }^{10}$ Ibid., Rêve du 28 mai 1964, pp. 353-355.

${ }^{11}$ Ibid., p. 355.

12 Ibid., $1^{\text {er }}$ avril 1965, p. 389.
} 
Le rêve du sourire associe d'ailleurs le travail du sourire - monde et moi - à celui de l'inspiration : «Il [le sourire] me possède, me distend, me remodèle comme l'inspiration. Il correspond en moi à ce désir, cet espoir, non pas de dire, mais d'être dit» (GM, p. 354).

Le monde, la matière psychique profonde, la vérité de l'expression poétique se révèlent être au fil du journal l'unique et triple « matière » qui constitue la « réalité » à laquelle l'écrivain a affaire ${ }^{13}$. C'est encore ce que résume le vœu du poète de "n'être plus qu'un contemplateur et un créateur $»^{14}:$ : [L]'abandon aux choses comme elles sont et la présence à ce qu'on fait» devient, à l'imitation de la petite Camille, la règle 《essentielle $»^{15}$. Le poète s'exhorte à « vivre » l'heure présente, dans toute sa plénitude sensible, au détriment même, parfois, de l'écriture: « $\mathrm{A}$ Chasté encore. Ne pas se préoccuper de noter, vivre seulement, jouir de cette heure merveilleuse où je suis rassemblé dans la beauté $»^{16}$.

Le corps et le monde, loin d'être une distraction coupable, sont progressivement reconnus comme une règle de vie qu'il faut adopter au prix d'une véritable ascèse. Être de plus en plus attentif au monde dans sa réalité est perçu comme un exercice quasiment vital, destiné à corriger une tendance dépressive :

Il y a toute une zone de vie plus élargie à laquelle j'ai parfois accès. Cette dimension est celle de la vie, de l'espoir, de la confiance. Lorsque j'en retombe c'est une sorte de mort, que j’ai vécue souvent.

Il me faut donc constamment m'élancer vers l'expansion, le contact, l'ouverture. Tout ce qui est repliement est affecté du

\footnotetext{
13 Sur un autre plan, Henry Bauchau utilise également des termes proches de ceux qu’il a pour dire l'heureuse maitrise du corps dans l'exercice sportif dominé quand il évoque le travail poétique accompli sans faillir ("Parmi les bonheurs non extatiques l'un des plus grands est celui de se posséder soi-même dans le cours d'un acte créateur. » [D86, 25 octobre 1963, p. 303]), ou décrit le poème "décanté et aminci, ne gardant qu'une structure de muscles » (Ibid., p. 192).

${ }^{14}$ GM, p. 288.

15 Ibid., 13 février 1964, p. 341.

${ }^{16}$ Ibid., 29 août 1962, p. 240.
} 
signe de la tristesse et de la mort. (GM, 12 octobre 1962, p. $248^{17}$ )

C'est aussi une façon, non pas de se détourner de l'exploration de l'expérience intérieure, mais d'éviter de s'y abstraire et de s'y perdre. Cette forme de sagesse, Henry Bauchau la place sous l'égide du philosophe Alain (et l'on pense à ses Propos sur le bonbeur):

Alain me montre que je me suis trop occupé de moi et pas assez du monde, de voir, saisir, sentir, entendre le monde.

C'est dans ce sens que je puis m'élargir encore énormément. (GM, 27 février 1962, p. $179^{18}$ )

Alors qu'Yves Bonnefoy rattache le risque de l'idéalisme et de la désincarnation de l'art à la tradition gréco-chrétienne et réfléchit d'emblée sur un plan philosophique et esthétique, Henry Bauchau, fidèle à son effort d'interprétation de la matière psychique, relie son travail d'apprentissage du monde à une dualité tout intime :

Ma nature comporte le choc entre deux pulsions divergentes, l'esprit de libération spirituelle, la démarche aérienne et l'exil solaire du Verseau et la force possessive, le goût de la terre et la sensualité du Taureau. (GM, 24 février 1960, p. 30.)

\section{«Ce qui compte, c'est d'être fidèle ${ }^{19}$ :} pour «l'écriture du corps ${ }^{20}$

La règle de vie se double d'une règle d'écriture. Il faut écrire juste, sans trahir la «matière » de l'expérience ni la « réalité » mise en jeu. Écrire est affaire de choix éthiques, et non pas seulement de principes esthétiques. Henry Bauchau se défie de ses « erreurs, particulièrement la grandiloquence et la liquidité sentimentale et formelle $»^{21}$, s'accuse d'une «tendance au gonflement tragique $»^{22}$. Écrire, comme l'y encourage la

\footnotetext{
${ }_{17}$ C'est en ce sens qu'il faut comprendre que l'univers romanesque puisse apparaitre " plus vrai que la vie » à Heny Bauchau, par opposition à l'existence aliénante qu'il lui semble mener quand il est pris par la routine à Montesano.

18 Voir aussi la note du 14 octobre 1962, p. 249, où Goethe est cité avec Alain.

${ }^{19}$ Ibid., 22 octobre 1962, p. 252.

${ }^{20} \mathrm{HD}$, p. 234.

${ }^{21}$ GM, 3 août 1960 , p. 77.

${ }^{22}$ Ibid., $1^{\text {er }}$ avril 1965 , p. 389.
} 
Sibylle, selon le «rythme intérieur », et non en suivant à un « rythme tout fait ${ }^{23}$, rester fidèle à sa propre voix, est, selon Henry Bauchau, une question d' "honneur», au sens tragique et classique du terme, quoique non sans humour, par opposition à la tentation de céder à des «critères de réussite extérieure ${ }^{24}$. C'est sur cette « véracité », associée à « la force de la sensation et de l'imagination du réel $»^{25}$ que le poète entend fonder son travail.

Comme l'a remarqué Catherine Mayaux, les journaux, chez Henry Bauchau, sont pour partie le laboratoire de la poésie et du roman ${ }^{26}$. Mais ils sont aussi le lieu où s'éprouve une écriture que le poète veut plus attentive au monde :

La lecture d'En toute candeur de Kenneth White a ravivé en moi le vieux goût des sensations brutes avec la matière et la nature. Je n'ai pas assez utilisé cela dans mes livres. C'est une des directions dans lesquelles je dois évoluer. [...]

$\mathrm{Ne}$ pas se contenter de percevoir des ensembles mais s'approcher au plus près, percevoir dans la neige le pied qui l'écrase différemment selon qu'elle est mouillée, fraîche, glacée, durcissante. Là encore beaucoup à faire. (GM, 25 octobre 1963, p. 302.)

Bien des notes de La Grande Muraille constituent ainsi de véritables exercices d'écriture et d'attention au réel. La description des paysages et des sensations éprouvées fait en outre souvent l'objet d'un travail de composition assez concerté. Les passages que nous avons cités plus haut répondent volontiers à un même schéma : mention de l'aspect admirable du paysage, évocation en crescendo des éléments du paysage, de leur beauté et du ravissement qu'ils suscitent, retombée dans le temps ordinaire. L'évocation assez brève des arbres dans la note du 25 octobre

${ }^{23}$ Ibid., 18 janvier 1960, p. 22. La Sibylle exhorte aussi Henry Bauchau à ne pas trahir, par une réconciliation factice, l'importance centrale, dans son histoire, de la « déchirure 》 (pp. 18, 21, 22).

${ }^{24}$ Ibid., 17 novembre 1961, p. 171.

${ }^{25}$ Ibid., 7 octobre 1960, p. 93.

${ }^{26}$ Catherine Mayaux, "La dictature du poème, ou le poème, cet inconnu. L'expérience poétique chez Henry Bauchau d'après ses journaux », dans Myriam Watthee-Delmotte (dir.), $N u(e), n^{\circ} 35$, mars 2007, pp. 199-211. 
1963 est même précédée d’une sorte de titre : «Les mélèzes » ${ }^{27}$. La note du 5 novembre 1961, propose le récit assez long d'une "grande promenade vers la Geltenhütte». Le poète, saisi par le froid et l'obscurité, se lance alors dans une ascension à la poursuite du soleil, " poussé par le désir du soleil et par une sorte de panique de l'ombre $»^{28}$. Tout le morceau est une sorte de réécriture, à la conclusion moins dramatique, de l'ascension de Jacques de Todi au chapitre deux du Monde désert de Jouve, dans «Bella Tola $»^{29}$. On pense encore au Rousseau des Rêveries quand Henry Bauchau trouve dans le paysage le point de départ de moments d'extase immanente où le sentiment de l'existence s'impose dans toute son intensité. Parfois, l'exercice d'écriture conduit paradoxalement à considérer le paysage de façon picturale, comme une composition, avec ses plans successifs, comme à Sils-maria, en août $1962^{30}$, ce qui est une façon de rompre avec le désir d'une appréhension «brute».

L'impératif de vérité qu'il se fixe incite en effet Henry Bauchau à repousser ce qui n'est pas au plus près de l'expérience telle qu'elle a été vécue, sans transposition symbolique («Ce n'est pas assez concret, je sors du lit profond, je déborde inutilement. C'est trop symbolique $\left.»^{31}\right) \mathrm{ni}$ commentaire («Il m'apparait de plus en plus que le goût de l'explication est le grand obstacle. Se rapprocher de la nudité, de l'image et du fait intérieur, lui laisser son abrupt $»^{32}$ ). Le passé ne doit donc pas donner lieu, pour Henry Bauchau, à une tentative de reconstitution nécessairement artificielle -, mais, grâce au travail de l'analyse, être relié, avec toutes les réappropriations dont il a pu faire l'objet au fil du temps, à la réalité de l'expérience présente : il faut «le voir réalisé, reconstruit dans une vision nouvelle ${ }^{33}$, « exprimer ce qu'est devenu en [soi] ce [qui a été ] vécu ${ }^{34}$ :

${ }^{27}$ Ibid., 25 octobre 1963, p. 302.

${ }^{28}$ Ibid., 5 novembre 1961, pp. 165-168.

${ }^{29}$ Pierre Jean Jouve, Euvre, Paris, Mercure de France, 1987, tome II, pp. 235-242.

${ }^{30}$ GM, 27 août 1962, pp. 236-237.

${ }^{31}$ Ibid., $1^{\text {er }}$ février 1961, p. 109.

32 Ibid., 4 mars 1962, p. 181. L'« image » a ici bien plus à voir avec l'impression reçue et

la marque qu'elle laisse dans l'imaginaire et la mémoire qu'avec le processus d'« excarnation » décrit par Yves Bonnefoy dans ses essais.

33 Ibid., 26 février 1960, p. 34.

${ }^{34}$ Ibid., 3 mars 1960, p. 35. 
L'analyse fait ressurgir le passé, le transforme et lui donne une physionomie et une signification nouvelles. Le passé continue, à partir de là, à vivre et à évoluer. Le temps passé est aussi un temps qui revient "modifié », un temps qui arrive. (GM, p. 36.)

Cette compréhension du rôle de l'analyse et du retour sur le passé permet à Henry Bauchau, alors même qu'il travaille à un livre du deuil, centré sur une « déchirure » fondamentale, d'échapper au sentiment de l'exil, au repli nostalgique et à l'idéalisation. Son écriture ne s'arrête pas à saisir un manque, une absence, mais une manière de vivre avec ou « dans plusieurs temps à la fois $»^{35}$, c'est-à-dire en affrontant la présence simultanée et les interactions de plusieurs strates de l'expérience et de la réalité. Et, plus largement, il faudrait selon Henry Bauchau essayer de saisir la réalité (intérieure-extérieure) dans le dynamisme de son surgissement et de ses transformations. Évoquant la relecture qu'il fait de l'œuvre de Proust, le poète se dit ainsi «infiniment plus proche de lui » en 1960 qu'à l'époque de sa première lecture :

J'avais à ce moment des préoccupations symboliques, un souci d'exprimer des idées et de donner un enseignement qui m'a en partie quitté. Moi aussi je suis de plus en plus attiré par la naissance de la sensation, par sa sincérité et son exactitude, compte tenu de l'abandon nécessaire à la venue des mots. (GM, 18 décembre 1960, pp. 103-104.)

Henry Bauchau semble ici rechercher une écriture qui, sans tenir de l'automatisme surréaliste, sans qu'il soit non plus précisé si elle est de nature pulsionnelle, préserverait une forme d'immédiateté.

Davantage encore, Henry Bauchau suggère une écriture qui serait comme en deçà de la représentation et des mécanismes du signe :

Une non-signification splendide devrait être possible, la nature en est pleine et le mouvement de l'art n'est-il pas de retrouver la nature non plus en la copiant, mais en la faisant naître comme elle fait? (GM, 14 janvier 1961, p. 108.)

35 Ibid., 22 avril 1960, p. 56. 
Ce souci d'« [ê]tre attentif à ce qui est là » et de «[m]éditer sans cesse sur le réel, le naturel, ce qui apparaît et qui signifie ${ }^{36}$ va dans ce même sens d'une signification immédiate, de l'ordre de la simple manifestation, purement phénoménologique. C’est en somme le désir - ou le rêve d'une écriture originelle, «l'écriture du corps » qui donne son titre à un poème de Célébration et qui serait "l'écriture antérieure qui brillait avant toutes les langues » et qui était parente de la «danse $»^{37}$.

Il faudrait aussi relire en ce sens le poème «Le petit jour » de La Chine intérieure où, à l'aube du jour anniversaire de ses soixante ans, le poète dit son amour de «la gloire des corps » et fait allusion à « la lumière d'avantnaitre / Avant les chambres et les prisons de la parole / Lorsque tout s'écrivait pour tous avec un corps $\gg{ }^{38}$. Le rapprochement s'impose avec le début de la note du 9 avril 1965 dans le journal, dont les termes sont très proches, et qui fait référence à la «beauté » et à la «gloire» de l'acte érotique :

Une des choses que l'âge abolit, peu à peu, c'est l'abandon à la gloire animale. Elle n'est pas seulement le déchaînement en soi des puissances animales mais la confiance que cela s'opère dans la beauté.

J'ai aimé la gloire du corps que je n'ai connue dans la pleine santé que par intervalles. Je ne l'ai pas trop aimée, je crois, car elle est une des plus fortes perceptions que nous puissions avoir de la plénitude. (GM, p. 393.)

Cette note aide sans doute à comprendre que «l'écriture du corps» serait celle qui, pour le poète, possèderait la plénitude et l'évidence d'un acte engageant amoureusement tout l'être dans la présence et l'unité. Il faudrait parvenir à une écriture qui ne soit plus que de l'ordre de la participation et de la communion. Les poèmes de la neige, d'« $[u] n$ lent poème corporel dans la matière de la neige ${ }^{39}$, dans La Chine intérieure, seraient sans doute à relire dans ce sens. C'est aussi ce qu'exprime la note du 2 novembre 1961. Le poète gravit le flanc de l'Eggli, «aimant[é] » par « un érable qui brille au soleil »:

\footnotetext{
${ }^{36}$ Ibid., 29 janvier 1964, p. 339.

${ }^{37} \mathrm{HD}$, p. 234.

${ }^{38}$ Ibid., p. 166.

${ }^{39}$ «Les mélèzes », HD, p. 189.
} 
Admirable beauté de l'érable auquel je parviens, son feuillage blond est adossé à une ligne sombre de sapins. Son tronc est chaud. Il ressemble à un divin danseur, tout en lui dit la splendeur de l'instant et jusqu'à cet essoufflement où je suis en m'adressant à lui. Il brille des derniers rayons du soleil et il va s'éteindre bientôt dans l'ombre. Il faudrait oser danser pour exprimer cela, faire une libation pour répondre à ce désir de don que le sien provoque en moi. (GM, pp. 164165.)

L'érable est pure manifestation, «tout en lui dit» la totalité de ce qui est, et le poète ne peut envisager de répondre à cette expression (au sens premier), qui est un «don» et une rencontre amoureuse, que par un don réciproque - danse et libation - qui l'engagerait tout entier.

La Grande Muraille est un journal d'une assez grande variété formelle : comptes rendus factuels, dialogues reconstitués, récits de rêve, souvenirs, descriptions, réflexions personnelles, transcriptions de poèmes en cours d'élaboration, notes sur l'écriture de La Déchirure... Mais, loin de s'y disperser, Henry Bauchau y poursuit obstinément l'identification, le développement et la réalisation de sa voix propre, en se confrontant aux deux questions fondamentales de l'écriture et de la «matière » de l'écriture. Esthétique, éthique, l'enjeu est aussi, en dernière analyse, profondément spirituel. L'écriture amoureuse dont le poète formule le vœu va de pair avec la «matière» qu'est la réalité intérieure-extérieure à laquelle il découvre qu'il doit se consacrer. Danser et faire libation à l'érable, c'est imaginer une écriture qui serait rituelle et liturgique, offerte à un « divin» dont Henry Bauchau écrit qu'il «ne le perçoi $[t]$ pas comme extérieur à [s]es propres états psychologiques, surtout à la perception de la beauté ou à une certaine présence de la vie cosmique. $»^{40}$. Ce sens d'un divin qui ne s'identifie plus à la transcendance traditionnelle, mais qui serait l'«horizon» même de l'expérience, son énigme, ce que Bauchau approche dans ses notes à chaque fois qu'il admire la beauté d'un paysage, inscrit assurément l'œuvre dans le renouveau lyrique de la seconde moitié $\mathrm{du} \mathrm{XX}^{\mathrm{e}}$ siècle et du début du $\mathrm{XXI}^{\mathrm{e}}$ siècle. Mais, quoique Jaccottet ou Bonnefoy restent pour lui les figures tutélaires d'une recherche qu'il partage, Henry Bauchau s'en éloigne pourtant dans la mesure où il les

${ }^{40}$ GM, 24 juillet 1964, p. 358. 
juge « obsédés par le mur de la mort » ${ }^{41}$. Celui qui écrit dans son journal que, "malgré les années, les masques que [lui] a imposés la vie, [il] ne ser[a] jamais qu'un enfant émerveillé » ${ }^{42}$ aurait sans doute trouvé davantage d'échos dans l'œuvre, inquiète mais habitée par la promesse et la joie d'un Mystère infiniment savoureux, d'un Jean-Claude Renard.

Aude Preta de Beaufort

Université de Lorraine

${ }^{41}$ Ibid., 18 novembre 1961, p. 172.

${ }^{42}$ Ibid., $1^{\text {er juillet 1962, p. } 212 .}$ 